\title{
EchoGéo
}

$57 \mid 2021$

Les enjeux de l'alimentation en eau potable des villes

\section{Le tourisme dans toute son épaisseur : un autre regard sur les espaces français}

Entretien avec Jean-Christophe Gay et Philippe Violier, réalisé par Serge Weber en visioconférence le 18 octobre 2021.

Jean-Christophe Gay, Philippe Violier et Serge Weber

\section{(2) OpenEdition}

Journals

Édition électronique

URL : https://journals.openedition.org/echogeo/22449

DOI : $10.4000 /$ echogeo.22449

ISSN : 1963-1197

Éditeur

Pôle de recherche pour l'organisation et la diffusion de l'information géographique (CNRS UMR 8586)

Référence électronique

Jean-Christophe Gay, Philippe Violier et Serge Weber, «Le tourisme dans toute son épaisseur : un autre regard sur les espaces français », EchoGéo [En ligne], 57 | 2021, mis en ligne le 15 octobre 2021, consulté le 05 novembre 2021. URL : http://journals.openedition.org/echogeo/22449 ; DOI : https:// doi.org/10.4000/echogeo.22449

Ce document a été généré automatiquement le 5 novembre 2021.

EchoGéo est mis à disposition selon les termes de la licence Creative Commons Attribution - Pas d'Utilisation Commerciale - Pas de Modification 4.0 International (CC BY-NC-ND) 


\section{Le tourisme dans toute son épaisseur : un autre regard sur les espaces français}

Entretien avec Jean-Christophe Gay et Philippe Violier, réalisé par

Serge Weber en visioconférence le 18 octobre 2021.

Jean-Christophe Gay, Philippe Violier et Serge Weber

\section{RÉFÉRENCE}

Philippe Violier, Philippe Duhamel, Jean-Christophe Gay, Véronique Mondou.

Le tourisme en France 1. Approche globale et 2. Approche régionale.

2021. Londres, ISTE, Série Tourisme et systèmes de mobilité, vol. 5 et 6, 273 et $221 \mathrm{p}$.

1 La parution de cet ouvrage sur une question qui semble à première vue classique, le tourisme en France, mérite d'être remarquée car en réalité, il y a très peu d'ouvrages de synthèse sur la question. De plus, les entrées, notions et thématiques proposées témoignent du profond renouvellement des approches et des concepts qui ont enrichi le champ des recherches sur le tourisme. L'originalité de nombreux exemples inattendus contribue à une lecture particulièrement savoureuse.

Serge Weber (SW). Cet ouvrage en deux volumes est un travail d'écriture collective. Pourriez-vous revenir sur sa genèse et les motivations qui vous ont amenés à vous lancer dans cette aventure?

Philippe Violer (PV). J'ai proposé à Philippe Duhamel, Véronique Mondou et à JeanChristophe Gay de faire un ouvrage assez global qui intègre les «confettis de l'empire ", donc nécessairement avec Jean-Christophe, spécialiste de ces espaces. Nous travaillons ensemble depuis presque trente ans, au gré de plusieurs livres et séminaires dans une véritable proximité. C'était l'occasion de nous retrouver. Nous souhaitions revenir sur une question qui est peu traitée : le dernier ouvrage sur le tourisme en France date de 1999, c'est celui de Nacima Baron. 
Jean-Christophe Gay (JCG). Auparavant, il n'y avait que celui, publié en 1993, de Daniel Clary. Or, il y a eu des avancées théoriques depuis lors, de nombreux outils et éclairages ont été mis au point au sein de l'équipe MIT, qui a été une formidable aventure (Knafou, 2018), que nous souhaitions mettre à jour pour le cas du tourisme en France.

PV. Les ouvrages précédents abordaient le tourisme comme une mobilité, des déplacements, sans véritablement poser la question du sens des pratiques touristiques et des lieux. En associant les voyages d'affaire, les pèlerinages, ils gardaient le cadre d'une définition très institutionnelle et n'ont pas déconstruit la définition portée par l'OMT (Organisation mondiale du tourisme) et par les institutions. Nous avions commencé à déconstruire les catégories pour comprendre le tourisme dans l'ouvrage collectif de 2002 Tourismes 1, Lieux communs (Équipe MIT, 2002). Et il faut tenir compte d'un élément important : le tourisme est un sujet qui est marginalisé dans les sciences sociales, en géographie notamment, ce qui est étonnant puisqu'il est profondément géographique. Il n'est pas considéré comme un objet sérieux ni comme un sujet noble - ce qui renvoie au débat sur les sujets nobles et les sujets vils. Il semblerait que la pandémie ait révélé l'importance économique du tourisme, ce n'était pas très bien vu auparavant, alors que nous en étions convaincus bien avant.

JCG. Le tourisme représente des centaines de milliers d'emplois, ce qui laisse imaginer qu'il y a de quoi être plus attentif aux recherches sur le tourisme. Il faut reconnaître que la géographie est mieux placée que les autres disciplines où les recherches et les chercheurs en tourisme sont rares et marginalisés. Une telle situation peut s'expliquer par un dédain généralisé pour le tourisme chez les élites économique ou intellectuelle, envisagé généralement sous l'angle de ses conséquences néfastes. Cette tourismophobie a été précisément disséquée par l'équipe MIT en 2002 et renvoie à notre difficile relation à la masse, à l'autre, au plaisir ou au repos, dans un monde occidental mettant fortement en avant l'individu et le travail. Ce n'est pas un hasard si nombre de formations universitaires en hôtellerie, restauration, accueil, patrimoine, événementiel, etc. sont entre les mains de géographes.

PV. C'est encore plus flagrant en économie, où le tourisme en tant qu'objet n'existe pas. On parle des marchés, des secteurs, certains disent que c'est un ensemble, alors que les manières de construire l'objet varient fortement.

JCG. C'est ce que nous avons souhaité montrer dans l'ouvrage : les fluctuations des délimitations de l'objet tourisme sont nombreuses et devaient être précisées.

SW. Je voulais vous inviter à revenir sur l'expérience de l'équipe MIT, sur la manière dont cette effervescence collective à contribuer à changer les regards.

PV. Les points théoriques qu'on a élaborés au MIT, au travers de productions véritablement pensées, discutées et écrites en commun, ont émergé autour de Rémy Knafou, qui a écrit une thèse en 1978 sur l'émergence des stations intégrées, ce qu'on a appelé des comptoirs, en haute altitude, comme Les Arcs ou La Plagne. Il a analysé ce type de lieu, ce qui était pour l'époque très novateur (Knafou, 1978).

JCG. Il avait été très critiqué à l'époque, notamment par Germaine Veyret, pour une thèse qui ne correspondait pas à l'approche géographique du moment. Ce n'était pas vu comme « un travail de géographe », car il ne portait pas sur un espace régional, 
mais sur un mode de production nouveau conduisant à rassembler un type de stations dispersés dans les Alpes françaises. Lorsque j'ai rejoint l'équipe, après avoir rencontré Rémy Knafou en 1990, j'avais fait partie, de 1987 à 1990, de l'équipe scientifique et technique de l'Atlas de la polynésie française (Dupon et al., 1993), alors que ma thèse ne portait pas sur le tourisme (Gay, 1992). J'ai intégré l'équipe en 1993-94, et je pourrais dire que ce qui nous a uni, c'est le point commun que nous avions, à savoir que les travaux qui étaient rédigés sur le tourisme ne nous convenaient pas. Rémy Knafou a proposé des pistes fécondes dans sa thèse, nous avons réfléchi pendant plusieurs années et développé une approche vraiment géographique du tourisme, attentive aux types de lieux, aux pratiques de mobilité, d'une manière différente de ce qui se faisait. Cette approche était fondée sur ce que nous avons nommé des tropismes, des catégories de lieux très déterminés par la nature. On oubliait le tourisme urbain à cette époque. Aux côtés de Rémy Knafou, il y avait déjà Philippe Duhamel, Florence Deprest qui avait fait sa thèse sous la direction de Rémy (Deprest, 1997), avec la collaboration très précieuse de Mireille Bruston qui était au laboratoire Intergéo. Et cette association prenait ses racines dans l'institut de Saint-Gervais, un groupe de recherche-action sur les stations alpines, dont on peut dire qu'il est l'ancêtre du MIT (Knafou, Bruston, Deprest, Duhamel, 1997).

PV. Pour ma part, je suis arrivé dans l'équipe en 1995. J'avais rencontré Rémy Knafou dans la commission de géographie du tourisme qu'il présidait - elle avait été créée bien avant, dans les années soixante-dix, par Michel Bonneau). J'étais à l'époque assez isolé à Angers, qui était alors une petite université avec peu de recherches sur le tourisme, et c'était l'occasion pour moi d'échanger avec d'autres collègues, d'autant plus que j'étais alors rattaché à une UMR de géographie sociale. Et pour la géographie sociale de l'époque, travailler sur le tourisme faisait de vous un extraterrestre. Le MIT était en tout point une équipe pertinente.

JCG. L'analyse du MIT avait pour fondement le constat de l'importance ignorée ou sous-estimée du tourisme. Si on veut retenir l'essentiel des apports du MIT, c'est la distinction entre tourisme et loisir, avec une réflexion très ouverte sur les autres disciplines. Nous sommes partis des lectures de Norbert Elias sur le processus de civilisation et d'Elias et Dunning sur la civilisation du sport comme violence maîtrisée, qui venait d'être traduit en français (Elias, Dunning, 1994 [1986]). La typologie des lieux touristiques, qui rompait avec les approches antérieures fondées sur les «milieux ", est aussi un résultat important de nos réflexions. Les démarches géohistoriques naissantes de Christian Grataloup nous ont aussi inspirés, pour réfléchir au modèle d'évolution des lieux touristiques et au concept de «moment de lieu », imaginé par Rémy Knafou pour comprendre l'émergence d'une nouvelle pratique touristique dans un lieu et la diffusion à la fois de celle-ci et du modèle de ce lieu, comme Nice et la Riviera avec l'invention de la « ville d'hiver ».

PV. Dans ce qu'Elias et Dunning appellent le "spectre des loisirs», qu'ils décomposent en trois catégories, ils situent, parmi la troisième, la plus efficace pour la déroutinisation, pour le relâchement des contraintes: "partir en vacances". C'était le point de départ de la réflexion. Le tourisme est un système dont le sens est la re-création des individus dans la société industrielle. C'est donc un élément central dans la construction de la civilisation industrielle, qui a justement fortement augmenté les contraintes. 
JCG. Or, à cette époque justement, le tourisme était abordé par l'offre et non par les individus.

PV. On a un peu renversé la perspective : considérer que les touristes sont au centre du tourisme. Ce sont les touristes eux-mêmes qui ont créé les premiers lieux du tourisme, là où il n'y avait rien, aucun hébergement, aucune infrastructure. On trouve dans l'ouvrage des photos de touristes, nous avons voulu mettre les touristes au centre du tourisme. Ce qu'ils font dans certains lieux, c'est fondamental pour comprendre pourquoi ces lieux sont devenus par la suite touristiques.

SW. Au sein de cette généalogie intellectuelle, je m'interrogeais sur l'influence qu'auraient pu avoir sur vos réflexions des travaux portant sur les pratiques de loisir des classes populaires, comme ceux de Patrick Champagne (1975) sur les vacances des agriculteurs, ou bien sûr des cultural studies britanniques, par exemple Richard Hoggart (1970).

PV. Oui, bien sûr, ces lectures ont compté, mais ces auteurs n'ont pas continué à travailler sur le tourisme. Mais on peut mentionner l'historien qui a travaillé sur "l'invention du mont Blanc", Philippe Joutard (1986). Il a proposé un concept que nous avons repris et retravaillé: le fait que les lieux ne sont pas seulement découverts, mais proprement inventés par les touristes.

SW. Dans cette veine, j'ai beaucoup apprécié à la lecture la manière dont vous déconstruisez complètement certains pans de ce que je croyais savoir d'incontestable et qu'il m'est arrivé de transmettre dans mes enseignements sans faire attention. Je pense en paticulier à ce que vous écrivez de très savoureux sur le plan Neige.

JCG. Oui, le plan Neige (ou les plans neige, entre 1964 et 1977) est assez typique de ces connaissances qui sont transmises d'un auteur à l'autre sans qu'on retourne aux sources pour les vérifier. Quand on creuse, on se pose la question de la signification réelle de ce que l'on nomme "Plan Neige ». En effet, si ce dernier fut bien réel, il remonte à 1970, soit plusieurs années après les travaux de construction de La Plagne (1962) et l'apparition d'autres stations comme Tignes, Vars, Avoriaz, Les Arcs, Isola 2000 ou Val Thorens. Le Plan Neige est en fait la forme paroxystique et tardive d'une doctrine qui s'infléchit dès l'année suivante, car dès l'hiver 1970-1971, la croissance de la pratique des sports d'hiver marque le pas. Ainsi, ce plan est baptisé a posteriori et s'infléchit fortement avec cette première crise. On le voit, l'expression Plan Neige est source de confusion. Il n'y a pas de réel plan d'aménagement à la différence de la MIACA (Mission interministérielle pour l'aménagement de la côte aquitaine, 1967-1988) et de la mission Racine (Mission interministérielle d'aménagement du littoral du Languedoc-Roussillon, 1963-1983). Il n’y a qu'à voir la desserte de la Tarentaise, qui concentre plusieurs stations de haute altitude (La Plagne, Les Arcs, Tignes, Val Thorens, Les Ménuires). Il a ainsi fallu attendre les jeux Olympiques d'Albertville en 1992 pour que soit construite une autoroute jusqu'à Albertville et une voie rapide la prolongeant jusqu'à Moûtiers et pour que les TGV arrivent à BourgSaint-Maurice.

PV. On peut évoquer un autre exemple: le modèle de R.W. Butler. Ce géographe écossais proposait, dans un article fondateur en 1980 dans la revue The Canadian Geographer, un modèle d'analyse du déclin des lieux touristiques. Pour simplifier: plus il y a de touristes dans un lieu, plus ce lieu va mal. Ce en quoi il a été novateur était qu'il a établi que les lieux touristiques sont créés par l'arrivée de touristes. C'était intéressant et pourtant, c'est passé relativement inaperçu à l'époque. Pour lui, quand le nombre de touristes augmente, cela pose des problèmes, qui vont se 
traduire par plusieurs évolutions possibles : le déclin, ou le rajeunissement. D'autres auteurs, comme l'Allemand Gormsen (1981) ou Jean-Marie Miossec (1977) n'ont retenu que le déclin. Cette annalyse s'est diffusée en étant réduite à l'expression "trop de touristes tue le tourisme ", qui est un lieu commun largement excessif. Au contraire, les cartes historiques qu'on peut trouver dans le volume de l'Atlas de France dirigé par Rémy Knafou (1997) montrent que de très anciennes stations touristiques comme Chamonix, Nice et d'autres sont toujours touristiques. Certes, quelques lieux ont cessé d'être touristiques et sont devenus autre chose, mais ce n'est pas une loi universelle.

JCG. R.W. Butler a assimilé une destination touristique à un produit. Il reprend la courbe en $\mathrm{S}$, utilisée en marketing, du cycle de vie d'un produit (depuis son élaboration jusqu'à son déclin et sa disparition). Il compare le cycle de vie d'un produit avec celui d'une station touristique, alors qu'en réalité, une station est beaucoup plus complexe. On pense bien sûr aux friches touristiques, mais il y en a peu, très peu! Elles existent surtout pour des raisons géopolitiques, dans un petit nombre de pays qui ont traversé des crises profondes (guerres civiles, occupation, partition, etc.).

PV. Et pourtant, R.W. Butler dit bien que pour qu'une station se relance, il faut qu'elle incorpore des innovations, il y a un rebond qui est possible, c'est très clair pour lui. Mais en reprenant son modèle, certains auteurs n'ont retenu que le déclin.

JCG. Le plus étonnant, c'est que le modèle de Butler est marginalement remis en question dans les travaux anglophones. C'est plutôt dans la géographie francophone qu'il est remis en cause et, aux yeux des géographes anglophones, nous passons pour des fous!

PV. Par ailleurs, Butler écrit "le lieu touristique»; au MIT, on a élaboré une typologie fondée sur deux grands types: d'une part, les lieux créés par le tourisme (stations et comptoirs), fragiles, effectivement, car spécialisés, mais moins au demeurant que les villes industrielles; d'autre part, les lieux investis par le tourisme (comme Paris) qui ne sont pas que touristiques et sont diversifiés, donc pour lesquels la disparition ou la régression du tourisme pose problème (par exemple avec la pandémie) mais qui continuent de fonctionner car ils disposent de plusieurs moteurs.

SW. À propos de friches touristiques, je pensais aux exemples développés par Alexandre Magnan (2008) à Saint-Martin.

JCG. Bien sûr, il se trouve qu'il est un de mes anciens doctorants. Oui, dans l'Outremer, les lois de défiscalisation ont gonflé artificiellement l'offre de la capacité hotelière en la déconnectant de la fréquentation, et inévitablement, la sortie de la défiscalisation a entraîné la fermeture d'hôtels. Certains sont restés en friche, mais la plupart non, ils ont été transformés en appartements. On retrouve la même chose en Nouvelle-Calédonie et en Polynésie française.

PV. Ce type de problème a été une de nos préoccupations pour le livre, qui concerne l'inadéquation récurrente de l'intervention de l'État pour le développement du tourisme. C'est l'objet du sixième chapitre (volume 1). Quand l'État intervient, c'est parfois à contresens. Par exemple, le complexe aquatique des Antilles de Jonzac est un cas éloquent. Le maire de Jonzac de l'époque, qui était président du conseil général de Charente-Maritime, a élaboré une politique touristique en partant du constat qu'il y avait trop de touristes sur le littoral et il qu'il valait mieux les envoyer 
vers l'intérieur des terres. On pourrait citer aussi le paléosite de Saint-Césaire dans le même département. Ces deux initiatives entendaient être des pièges à touristes, mais ce sont avérés être des fours, comme d'autres qui ont été projetées dans des PDZR (programmes de développement des zones rurales). Au bout d'un moment, le groupe Pierre \& Vacances s'est retiré de Jonzac, et le paléosite qui n'a jamais été rentable, est aujourd'hui sous perfusion des finances du département. On pourrait en citer un troisième, le pôle mécanique de la Haute-Saintonge à La Genetouze : lorsque j'ai posé la question dans mes enquêtes à des personnes du Conseil général, elles ne savait même pas où c'était! On observe que les politiques souhaitent inaugurer des sites culturels, mais pas des sites touristiques. Par exemple, le musée du pont du Gard est une catastrophe financière. Les élus veulent inaugurer des lieux culturels, c'est mieux, on pourrait citer plusieurs exemples d'intervention politique malencontreuse. Le rôle de l'institutionnel c'est aussi la volonté d'imposer les interêts du territoire administratif à une pratique sociale qui n'en a cure.

SW. Les grands sites de France sont du même ordre?

PV. Il y a une série de pratiques qui sont vues comme de «bons tourismes»: la culture, le sport (« les plages actives, dynamiques»), et un discours sur le « mauvais » tourisme (la sieste ou la pétanque par exemple). Pour la plupart des grands sites, il s'agit plutôt d'une logique d'intervention d'aménagement qui n'est pas toujours mal faite, et même assez souvent pertinente, mais qui se fait après coup : le lieu est déjà très fréquenté, et la fréquentation entraîne une dégradation, une usure, il devient nécessaire d'intervenir pour rétablir les paysages et améliorer l'accueil. La pointe du Raz en est l'exemple le plus connu.

SW. Est-ce que les recherches sur les enjeux du tourisme dans les pays du Sud, avec leur proximité avec la critique post- ou décoloniale, vous semblent avoir joué un rôle dans la manière d'aborder le tourisme dans un contexte comme celui de la France?

PV. Le tourisme dans les pays du Sud a d'abord été traité à travers le modèle centre/ périphérie avec des travaux comme ceux de Georges Cazes (1989), spécialiste du tiersmonde, qui pointait du doigt la domination que reproduisaient les «nouvelles colonies de vacances ", avec ce jeu de mot. Depuis, cette question a été profondément retravaillée, notamment par Isabelle Sacareau (1997), au sein de l'équipe MIT, qui a montré au contraire que le tourisme au Népal avait été saisi par les sherpas pour leur reproduction sociale alors que leur survie économique était très menacée au début des années 1950 en raison de la fermeture de la frontière chinoise suite à l'arrivée de Mao au pouvoir et de l'indépendance de l'Inde, à un moment où le Népal était en pleine explosion démographique. Le sociologue Michel Picard (1993) montrait la même chose pour Bali. Le discours global condamnait le tourisme, mais les auteurs qui étaient sur le terrain ont montré que c'était plus complexe : dans de nombreux cas, les sociétés locales ont su s'approprier le tourisme à leur avantage. Car contrairement à un usage commun du terme, le tourisme n'est pas un acteur, c'est un agent. Parfois les habitants s'en saisissent alors que dans d'autres situations, le tourisme leur est clairement imposé. Pour ce dernier cas de figure, on peut citer l'exemple du Club Méditerranée au Sénégal : le président Senghor a fait un véritable un pont d'or à l'entreprise pour s'établir en Casamance, parce qu'il était wolof casamançais.

JCG. L'approche postcoloniale montre bien l'influence des sociétés locales sur le tourisme, elle permet de réévaluer leur rôle et leur action sur le tourisme. Georges 
Cazes avait apporté des choses intéressantes, mais aujourd'hui, les approches se sont enrichies et on ne peut pas se contenter de ce discours propre aux années 1980. Pour rester en Indonésie, Sylvine Pickel-Chevalier (2017), qui a elle aussi travaillé sur Bali, montre la même chose. Elle analyse la manière dont les Balinais s'approprient euxmêmes le tourisme. Certaines sociétés émergentes sont devenues touristiques par elles-mêmes.

SW. Dans votre ouvrage, on est frappé par votre attention constante à I'historicisation, au temps long, à la genèse des lieux ou des pratiques, cela contribue à mon avis à la grande originalité et à l'intérêt de ce livre.

PV. L'exemple le plus intéressant est celui de Nice et de la Côte d'Azur, traité par Jean-Christophe, qui montre comment le tourisme a permis à une petite ville assez pauvre de devenir une véritable métropole.

JCG. En effet, le cas niçois est très éloquent: dès le début de la fréquentation touristique à Nice, ce qui est frappant, c'est comment les interactions entre la société locale et les premiers étrangers, les Anglais en particulier, ont fait de la ville une métropole à dimension internationale, avec les différents capitaux qui se sont accumulés en deux siècles et demi. C'est la spécificité de Nice, cette combinaison de capitaux économiques, culturels, migratoires, diplomatiques, sportifs, etc., une accumulation qui fait de la Côte d'Azur un modèle pour d'autres. Quand on lit les géographes sur la géographie de la France, il est rare d'en trouver qui comprennent vraiment la Côte d'Azur : on ne peut pas la comprendre avec des critères classiques, tels que l'urbanité, qui ne fonctionnent pas pour ce type de lieux. J'ajoute que j'ai mené pour ce livre une analyse inédite de l'histoire du tourisme en outre-mer.

PV. Dans notre choix de plan pour le livre, nous avons insisté pour traduire cela dans l'approche régionale concernant l'hexagone - c'est un peu différent dans la partie sur l'outre-mer. Nous avons tenu à faire une régionalisation spécifiquement touristique, refusant les découpages classiques, par milieux ou selon le maillage administratif. Nous distinguons les lieux créés par le tourisme (les lieux nouveaux, les comptoirs) et les mondes pleins investis par le tourisme. C'est pourquoi pour traduire l'exemplarité du cas niçois, il est d'abord abordé dans la première catégorie (chapitre 1) puis passe dans le chapitre 2, en seconde position juste après Paris, avant les autres villes. Je me souviens d'une soutenance de thèse où un membre du jury avait exprimé sa gêne d'entendre qu'on pouvait identifier des régions touristiques, c'était très surprenant.

PV. Les pratiques touristiques sont centenaires, ce n'est pas un effet de mode. L'apparition des bains chauds et des corps exposés remonte au début du $\mathrm{XX}^{\mathrm{e}}$ siècle, qui remplacent le bain froid et le corps caché qui étaient la norme depuis le milieu XVII siècle. Il est fondamental d'avoir ce temps long à l'esprit.

JCG. Nous avons voulu restituer l'épaisseur touristique, car on ne comprend pas un lieu touristique sans comprendre comment il se transforme. C'est un des axes de recherche du nouvel Institut du tourisme Côte d'Azur, que je dirige.

PV. Alors même que peu d'historiens travaillent sur le tourisme. On pourra citer Laurent Tissot sur la genèse de l'industrie touristique en Suisse (2000) et évidemment, Marc Boyer qui a écrit des ouvrages fondamentaux, notamment sur l'invention du tourisme (1996). On peut dire qu'il est le premier historien du tourisme, son travail sur l'invention de la Côte d'Azur est passionnant, mais il n'a pas fait carrière. 
SW. Vous avez réussi à conjuguer le renouvellement épistémologique et théorique des études sur le tourisme avec une profusion d'exemples originaux. Vous intégrez des cas d'étude très récents tout en maintenant une attention géohistorique permanente. On peut donc dire que cet ouvrage satisfait à tout ce qu'on peut attendre d'une véritable mise à jour sur un sujet aussi important. Un très grand merci à vous deux et à vos deux co-auteurs.

\section{BIBLIOGRAPHIE}

Baron-Yellès, N., 1999. Le tourisme en France, territoires et stratégies. Paris, Armand Colin.

Boyer M., 1996. L'invention du tourisme. Paris, Gallimard.

Butler, R. W., 1980. The concept of a tourist area cycle of evolution: implications for management of resources. The Canadian Geographer, $\mathrm{n}^{\circ}$ 24, p. 5-12.

Cazes G., 1989. Les Nouvelles Colonies de vacances? Le tourisme international à la conquête du Tiers Monde. Paris, L'Harmattan.

Champagne P., 1975. Les paysans à la plage. Actes de la recherche en sciences sociales, $\mathrm{n}^{\circ} 1-2, \mathrm{p}$. 21-24.

Clary D., 1993. Le tourisme dans l'espace français. Issy-les-Moulineaux, Masson.

Deprest F., 1997. Enquête sur le tourisme de masse. L'écologie face au territoire. Paris, Belin.

Dupon J.-F., Bonvallot J., Vigneron E. (dir.), 1993. Atlas de la Polynésie Française. Paris, ORSTOM.

Elias N., Dunning E., 1994 [1986]. Sport et civilisation. La violence maîtrisée. Paris, Fayard.

Équipe MIT, 2002. Tourismes 1. Lieux communs. Paris, Belin.

Gay J.-C., 1992. L'étendue, les lieux et l'espace géographique pour une approche du discontinu. Thèse de doctorat en géographie, Université Aix-Marseille 2.

Gormsen E., 1981. The Spatiotemporal Development of International Tourism. Attempt at the Centre-Periphery Model. In Baretje R. (dir.), La consommation d'espace par le tourisme et sa préservation. Aix-en-Provence, CHET, p. 150-170.

Hoggart R., 1970. La Culture du pauvre. Étude sur le style de vie des classes populaires en Angleterre. Paris, Seuil.

Joutard P. (éd.), 1986. L'invention du mont Blanc. Paris, Gallimard/Julliard.

Knafou R., 1978. Les stations intégrées de sports d'hiver dans les Alpes françaises. Paris, Masson.

Knafou R. (dir.), 1997. Atlas de France Vol. 7 : Tourisme et loisirs. Montpellier, Paris, GIP RECLUS, La Documentation française.

Knafou R., 2018. L'expérience de l'Équipe MIT (Mobilités, Itinéraires, Tourismes), premier groupe français de recherche sur le tourisme : une aventure humaine et intellectuelle. Bulletin de l'association de géographes français, vol. 95, $\mathrm{n}^{\circ}$ 4, p. 455-467.

Knafou R., Bruston M., Deprest F., Duhamel P., 1997. L'institut de Saint-Gervais. Recherche action dans la montagne touristique, Paris, Belin. 
Magnan A., 2008. L'espace littoral a-t-il toujours de la valeur ? Réflexion à partir du cas de l'île de Saint-Martin (Petites Antilles). Norois, ${ }^{\circ}$ 206, p. 37-52.

Miossec J.-M., 1977. Un modèle de l'espace touristique. L'Espace géographique, vol. 1, p. 41-48.

Picard M., 1993. Bali. Tourisme culturel et culture touristique. Paris, L'Harmattan.

Pickel-Chevalier S., 2017. Tourism in Bali and the Challenge of Sustainable Development. Cambridge, Cambridge Scholars Publishing.

Sacareau I., 1997. Porteurs de l'Himalaya : le trekking au Népal. Paris, Belin.

Tissot L., 2000. Naissance d'une industrie touristique. Les Anglais et la Suisse au XIX ${ }^{e}$ siècle. Lausanne, Éditions Payot

\section{INDEX}

Thèmes : Sur l'Écrit

\section{AUTEURS}

\section{JEAN-CHRISTOPHE GAY}

Jean-Christophe Gay est professeur des universités à l'IAE Nice, Université Côte d'Azur, et directeur scientifique de l'Institut du tourisme Côte d'Azur. Il a notamment publié :

- Gay J.-C., 2012. Why Is Tourism Doing Poorly in Overseas France? Annals of Tourism Research, vol. 39, p. 1634-1652.

- Gay J.-C., Decroly J.-M., 2018. Les logiques de la diffusion du tourisme dans le monde : une approche géohistorique. L'Espace géographique, vol. 47, n² 2, p. 102-120.

- Gay J.-C., 2021. La France d'outre-mer. Terres éparses, sociétés vivantes. Paris, Armand Colin, 285 p.

\section{PHILIPPE VIOLIER}

Philippe Violier est professeur émérite à l'Université d'Angers, membre de l'UMR ESO 6590. Il a notamment publié :

- Violier P., 2016. La troisième révolution touristique. Mondes du Tourisme [En ligne], Hors-série. URL: http://journals.openedition.org/tourisme/1256 - DOI: https://doi.org/10.4000/tourisme. 1256

- Stock M., Coëffé V., Duhamel P., Violier P., 2020 [2017]. Les enjeux contemporains du tourisme. Une approche géographique. Presses Universitaires de Rennes, $500 \mathrm{p}$.

- Violier P., Taunay B., 2019. Les lieux touristiques du Monde. De la mondialisation à la mondialité. Londres, ISTE Editions, $322 \mathrm{p}$. 